\title{
Signed cycle double covers
}

\author{
Lingsheng Shi* Zhang Zhang \\ Department of Mathematical Sciences \\ Tsinghua University \\ Beijing, 100084, China \\ lshi@math.tsinghua.edu.cn
}

Submitted: Jan 20, 2017; Accepted: Dec 10, 2018; Published: Dec 21, 2018

(C) The authors. Released under the CC BY-ND license (International 4.0).

\begin{abstract}
The cycle double cover conjecture states that every bridgeless graph has a collection of cycles which together cover every edge of the graph exactly twice. A signed graph is a graph with each edge assigned by a positive or a negative sign. In this article, we prove a weak version of this conjecture that is the existence of a signed cycle double cover for all bridgeless graphs. We also show the relationships of the signed cycle double cover and other famous conjectures such as the Tutte flow conjectures and the shortest cycle cover conjecture etc.
\end{abstract}

Mathematics Subject Classifications: 05C21, 05C22, 05C38

\section{Introduction}

The Cycle Double Cover Conjecture is a famous conjecture in graph theory. It states that every bridgeless graph has a collection of cycles which together cover every edge of the graph exactly twice. Let $G$ be a graph. A collection of cycles of $G$ is called a cycle cover if it covers each edge of $G$. A cycle double cover of $G$ is such a cycle cover of $G$ that each edge lies on exactly two cycles. A graph is called even if its vertices are all of even degree. A $k$-cycle double cover of $G$ consists of $k$ even subgraphs of $G$. This conjecture is a folklore (see [3]) and it was independently formulated by Szekeres [23], Itai and Rodeh [9], and Seymour [22]. In fact, Szekeres raised this conjecture just for cubic graphs, while Seymour put it in a general case.

Conjecture 1. (The Cycle Double Cover Conjecture $[22,23]$ ) Every bridgeless graph has a cycle double cover.

*Project 11771246 supported by National Natural Science Foundation of China. 
Preissmann [19] and Celmins [5] independently proposed the following stronger conjecture.

Conjecture 2. [5, 19] Every bridgeless graph has a 5-cycle double cover.

The cycle cover problem is closely related to the flow theory. Let $G=(V, E)$ be a graph. An integer flow of $G$ is an ordered pair $(D, f)$ satisfying

$$
\sum_{s(e)=v} f(e)=\sum_{t(e)=v} f(e) \quad \forall v \in V
$$

where $D$ is an orientation of $G$ and $f$ is a function $E \mapsto \mathbb{Z}, s(e)$ denotes the source of $e$ and $t(e)$ denotes the tail of $e$. A nowhere-zero $k$-flow of $G$ is an integer flow such that $0<|f(e)|<k$ for every edge $e$ of $G$. A group flow is defined similarly by taking values in an Abelian group instead of $\mathbb{Z}$. For instance, if a flow takes values in the finite cyclic group $\mathbb{Z}_{k}$, then we call it a $\mathbb{Z}_{k}$-flow. The support of a flow $f$, denoted by $\operatorname{supp}(f)$, is the set of all the edges $e$ of $G$ with $f(e) \neq 0$. Tutte [24] found the following relationship of the integer flow and the group flow.

Theorem 3. [24] A bridgeless graph admits a nowhere-zero $k$-flow if and only if it admits a nowhere-zero group flow with group order $k$.

Generalizing the coloring problem of planar graphs, Tutte proposed the following three famous conjectures on integer flows.

Conjecture 4. (The Five-Flow Conjecture [24]) Every bridgeless graph admits a nowherezero 5 -flow.

Conjecture 5. (The Four-Flow Conjecture [25]) Every bridgeless graph containing no subdivision of the Petersen graph admits a nowhere-zero 4-flow.

Conjecture 6. (The Three-flow conjecture [4]) Every bridgeless graph without 3-edge cuts admits a nowhere-zero 3-flow.

Jaeger [12] and Seymour [20] derived two fundamental theorems on integer flows, the Eight-Flow Theorem and the Six-Flow Theorem.

Theorem 7. (The Eight-Flow Theorem [12]) Every bridgeless graph admits a nowherezero 8-flow.

Theorem 8. (The Six-Flow Theorem [20]) Every bridgeless graph admits a nowhere-zero 6-flow.

The following relationship of cycle double covers and flows can be found in [11].

Theorem 9. [11] A graph has a 4-cycle double cover if and only if it admits a nowherezero 4-flow. 
For more backgrounds on cycle covers and flows we refer to the monographs [26, 27]. An ordered pair $(G, \omega)$ is called a weighted graph if $\omega: E(G) \mapsto \mathbb{Z}$ is an integer-valued function which is called the weight. Thus a graph $G$ can be viewed as a special weighted graph $(G, \omega)$ with the constant weight $\omega=1$. Let $G_{i}=\left(G, \omega_{i}\right)$ be a weighted graph for $i=1,2$. The sum of $G_{1}$ and $G_{2}$ is defined by

$$
G_{1}+G_{2}=\left(G, \omega_{1}+\omega_{2}\right),
$$

where $\left(\omega_{1}+\omega_{2}\right)(e)=\omega_{1}(e)+\omega_{2}(e)$ for each $e \in E(G)$. The difference of $G_{1}$ and $G_{2}$ is defined by

$$
G_{1}-G_{2}=\left(G, \omega_{1}-\omega_{2}\right),
$$

where $\left(\omega_{1}-\omega_{2}\right)(e)=\omega_{1}(e)-\omega_{2}(e)$ for each $e \in E(G)$.

A general signed graph $(G, \omega)$ is such a weighted graph that $\omega(e) \in\{0, \pm 1\}$ for all $e \in E(G)$. This generalizes the notion of signed graph which edges are allowed to take only two values \pm 1 . Since the support of a general signed graph is just its signed subgraph, we simply call it a signed graph without confusion. Thus a subgraph $H$ of $G$ can be viewed as a signed graph $\left(G, \omega_{H}\right)$, where $\omega_{H}(e)=1$ for $e \in E(H)$ and $\omega_{H}(e)=0$ for $e \in E(G) \backslash E(H)$. A signed graph $(G, \omega)$ is even if $\sum_{u \in N(v)} \omega(u v)$ is even for all $v \in V(G)$, where $N(v)$ denotes the neighborhood of $v$ in $G$. An edge $e$ of $G$ is called negative if $\omega(e)=-1$. The number of negative edges in $(G, \omega)$ is $\left|\omega^{-1}(-1)\right|$.

A collection of even signed graphs $\left\{G_{1}, G_{2}, \ldots, G_{t}\right\}$ of $G$ is called a signed cycle double cover of $G$ if it satisfies

$$
\sum_{i=1}^{t} \omega_{G_{i}}(e)=2, \quad \forall e \in E(G) .
$$

A $k$-signed cycle double cover consists of $k$ even signed graphs. Let $\Gamma$ be a signed cycle double cover. The total number of negative edges of $\Gamma$, denoted by $\nu(\Gamma)$, is defined by

$$
\nu(\Gamma)=\sum_{H \in \Gamma}\left|\omega_{H}^{-1}(-1)\right|
$$

Though Conjectures 1 and 2 are widely open and it is well known that the Petersen graph has no 4-cycle double cover, we establish the following weak version by replacing cycles with signed cycles. This is analogous in some sense to a result of Seymour [21] who proved the weak signed versions of the Berge-Fulkerson Conjecture [8, 21] about double covers by perfect matchings and the Tutte Four-Flow Conjecture in its dual coloring form for cubic graphs.

Theorem 10. Every bridgeless graph of order $n$ and size $m$ has a 4-signed cycle double cover $\Gamma$ with $\nu(\Gamma) \leqslant \min \{n / 3, m / 9\}$.

Máčajová and Škoviera [18, Theorem 1.1] proved that every bridgeless cubic graph has a Fano coloring which uses at most six different lines. Moreover, if at most six points are used in the Fano coloring, then there is a nowhere-zero 4-flow. This is a consequence of the following theorem which is stated in the flow form instead of coloring. 
Theorem 11. A graph admits a nowhere-zero 4-flow if and only if it admits a nonsurjective nowhere-zero $\mathbb{Z}_{2}^{3}$-flow.

Though the bound in Theorem 10 is hardly improved (see Corollary 18), it may not be sharp because of the following result.

Theorem 12. There exist infinitely many graphs of size $m$ such that all 4-signed cycle double covers have at least $m / 15$ negative edges.

In view of Theorems 10 and 12, we propose the following conjecture.

Conjecture 13. Every bridgeless graph of size $m$ has a 4 -signed cycle double cover with at most $m / 15$ negative edges.

Note that Theorem 10 implies Conjecture 13 for all bridgeless graphs of order $n$ and size $m \geqslant 5 n$. We prove Theorems 10, 11, and 12 in Section 2 and finish with a discussion in Section 3 on the relationship of the signed cycle double cover and other conjectures, such as the Shortest Cycle Cover Conjecture and the Tarsi Conjecture on the shortest 3 -cycle covers etc.

\section{Proofs of main results}

The proof of Theorem 10 is based on the Six-Flow Theorem and the Eight-Flow Theorem. It is to construct directly a signed cycle double cover which differs from the approach of Seymour [21] who made signed covers by perfect matchings indirectly from multicolorings. The idea of our proof is as follows.

A 6 -flow induces a $\mathbb{Z}_{3} \times \mathbb{Z}_{2}$-flow. The support $C$ of the $\mathbb{Z}_{2}$-flow can be taken as a disjoint union of circuits with size at most $n$. The $\mathbb{Z}_{3}$-flow induces a 3 -cycle double cover of its support. All even subgraphs form an Abelian group under the operation of symmetric difference, and all even weighted graphs also form an additive group, though all even signed graphs do not. Applying the three operations of addition, subtraction, and symmetric difference to $C$ and the three cycles, and taking care of the weights of all edges, we get two 4 -signed cycle double covers with disjoint sets of negative edges $E_{1}$ and $E_{2}$ appearing only on the support $C$. The three disjoint sets $E_{1}, E_{2}$, and $E(C) \backslash E_{1} \cup E_{2}$ form a partition of all edges of $C$. But both $E_{1}$ and $E_{2}$ might be of size greater than $n / 3$. In this case, we make a new $\mathbb{Z}_{3}$-flow by pivoting the $\mathbb{Z}_{2}$-flow so that one of the two sets $E_{1}$ and $E_{2}$ changes to the set $E(C) \backslash E_{1} \cup E_{2}$ which is of size at most $n / 3$.

An 8-flow induces a $\mathbb{Z}_{2}^{3}$-flow that in turn induces a partition of all edges into 7 subsets. The three supports of the $\mathbb{Z}_{2}$-flows all induce even subgraphs. Taking symmetric differences of the three supports yields four new even subgraphs. The key observation is that every one of the 7 subsets of edges is covered exactly 4 times by the 7 even subgraphs and the corresponding 4 even subgraphs form a signed cycle double cover. Bermond et al. [2] observed that the $\mathbb{Z}_{2}^{3}$-flow can be taken so that one of the supports is large with at least $2 \mathrm{~m} / 3$ edges. Three of the 7 subsets of edges are disjoint with the largest support, so we can take the smallest one as the set of negative edges which size turns out be at most $m / 9$. 
Proof of Theorem 10. Let $G$ be a bridgeless graph of order $n$ and size $m$. We first show that $G$ has a 4 -signed cycle double cover with $\nu \leqslant n / 3$. By Theorem 3 and the Six-Flow Theorem, the graph $G$ admits a nowhere-zero $\mathbb{Z}_{3} \times \mathbb{Z}_{2}$-flow, say $(f, g)$. Let $C$ be the even subgraph of $G$ induced by $\operatorname{supp}(g)$. By the proof of the Six-Flow Theorem [20], the flow $g$ can be selected such that $C$ is a union of disjoint circuits with $|E(C)| \leqslant n$. Note that $f$ is a nowhere-zero $\mathbb{Z}_{3}$-flow on $\operatorname{supp}(f)$ which can be turned to a nowhere-zero 3 -flow by Theorem 3. Since a 3-flow can be viewed as a 4-flow, the support of $f$ has a 3-cycle double cover, denoted by $\left\{C_{01}, C_{02}, C_{03}\right\}$. Indeed, if the pair $\left(g_{1}, g_{2}\right)$ is a nowhere-zero $\mathbb{Z}_{2}^{2}$-flow on $\operatorname{supp}(f)$, then let $C_{01}$ and $C_{02}$ be the even subgraphs induced by $\operatorname{supp}\left(g_{1}\right)$ and $\operatorname{supp}\left(g_{2}\right)$ respectively, and let $C_{03}=C_{01} \triangle C_{02}$ be the subgraph of $G$ induced by the symmetric difference $E\left(C_{01}\right) \triangle E\left(C_{02}\right)$.

Let $f_{i}=f+i g(\bmod 3)$ for $i=1,2$. As for the flow $f$, both $\operatorname{supp}\left(f_{1}\right)$ and $\operatorname{supp}\left(f_{2}\right)$ also have 3 -cycle double covers, denoted by $\left\{C_{11}, C_{12}, C_{13}\right\}$ and $\left\{C_{21}, C_{22}, C_{23}\right\}$, respectively. One can easily verify that

$$
C_{1}=C \triangle C_{11}+C \triangle C_{12}+C \triangle C_{13}-C_{01}-C_{02}-C_{03}
$$

is a signed even graph of $G$. Indeed, let $e$ be an edge of $G$ and we go through the following three cases.

Case 1. $f(e)=0, g(e) \neq 0$.

In this case, we have $e \in E(C) \cap \operatorname{supp}\left(f_{1}\right) \backslash \operatorname{supp}(f)$, and then $e$ is in exactly two of $\left\{C_{11}, C_{12}, C_{13}\right\}$ and none of $\left\{C_{01}, C_{02}, C_{03}\right\}$. Thus $e$ is in only one of

$$
\left\{C \triangle C_{11}, C \triangle C_{12}, C \triangle C_{13}\right\}
$$

and $\omega_{C_{1}}(e)=1$.

Case 2. $f(e) \neq 0, g(e)=0$.

In this case, we have $e \in \operatorname{supp}(f) \cap \operatorname{supp}\left(f_{1}\right) \backslash E(C)$, and then $e$ is in exactly two of $\left\{C_{i 1}, C_{i 2}, C_{i 3}\right\}$ for $i=0,1$. Thus $e$ is in exactly two of $\left\{C \triangle C_{11}, C \triangle C_{12}, C \triangle C_{13}\right\}$ and $\omega_{C_{1}}(e)=0$.

Case 3. $f(e) \neq 0, g(e) \neq 0$.

If $f(e)=1$, then $e \in E(C) \cap \operatorname{supp}(f) \cap \operatorname{supp}\left(f_{1}\right)$ and thus $e$ is in exactly two of $\left\{C_{i 1}, C_{i 2}, C_{i 3}\right\}$ for $i=0,1$ and in only one of $\left\{C \triangle C_{11}, C \triangle C_{12}, C \triangle C_{13}\right\}$ which implies that $\omega_{C_{1}}(e)=-1$. If $f(e)=2$, then $e \in E(C) \cap \operatorname{supp}(f) \backslash \operatorname{supp}\left(f_{1}\right)$ and thus $e$ is in exactly two of $\left\{C_{01}, C_{02}, C_{03}\right\}$ and in all of $\left\{C \triangle C_{11}, C \triangle C_{12}, C \triangle C_{13}\right\}$ which implies that $\omega_{C_{1}}(e)=1$.

The even signed graph $C_{1}$ together with the three even subgraphs $C \triangle C_{21}, C \triangle C_{22}$, $C \triangle C_{23}$ forms a 4 -signed cycle double cover of $G$, denoted by $\Gamma_{1}$, which is shown in Table 1 . Similarly, let

$$
C_{2}=C \triangle C_{21}+C \triangle C_{22}+C \triangle C_{23}-C_{01}-C_{02}-C_{03},
$$

which together with the three even subgraphs $C \triangle C_{11}, C \triangle C_{12}, C \triangle C_{13}$ forms another 4-signed cycle double cover of $G$, denoted by $\Gamma_{2}$, see Table 2 . 


\begin{tabular}{cc|ccccc|c}
$g(e)$ & $f(e)$ & $f_{1}(e)$ & $f_{2}(e)$ & $\omega_{C}(e)$ & $\omega_{C_{1}}(e)$ & $\omega_{C \triangle C_{21}+C \triangle C_{22}+C \triangle C_{23}}(e)$ & $\sum_{H \in \Gamma_{1}} \omega_{H}(e)$ \\
\hline 0 & 1 & 1 & 1 & 0 & 0 & 2 & 2 \\
1 & 0 & 1 & 2 & 1 & 1 & 1 & 2 \\
1 & 1 & 2 & 0 & 1 & -1 & 3 & 2 \\
1 & 2 & 0 & 1 & 1 & 1 & 1 & 2
\end{tabular}

Table 1: The 4-signed cycle double cover $\Gamma_{1}$.

\begin{tabular}{cc|ccccc|c}
$g(e)$ & $f(e)$ & $f_{1}(e)$ & $f_{2}(e)$ & $\omega_{C}(e)$ & $\omega_{C_{2}}(e)$ & $\omega_{C \triangle C_{11}+C \triangle C_{12}+C \triangle C_{13}}(e)$ & $\sum_{H \in \Gamma_{2}} \omega_{H}(e)$ \\
\hline 0 & 1 & 1 & 1 & 0 & 0 & 2 & 2 \\
1 & 0 & 1 & 2 & 1 & 1 & 1 & 2 \\
1 & 1 & 2 & 0 & 1 & 1 & 1 & 2 \\
1 & 2 & 0 & 1 & 1 & -1 & 3 & 2
\end{tabular}

Table 2: The 4-signed cycle double cover $\Gamma_{2}$.

Note that only in Case 3 can negative edges occur in $C_{1}$ or $C_{2}$ and all such negative edges are on $C$. Indeed, an edge $e$ in $C_{1}$ is negative if and only if $f(e)=g(e)=1$ and an edge $e^{\prime}$ in $C_{2}$ is negative if and only if $f\left(e^{\prime}\right)=2$ and $g\left(e^{\prime}\right)=1$.

Let $E_{i}=f^{-1}(i) \cap E(C)$ for $i=0,1,2$. Clearly, $\nu\left(\Gamma_{i}\right)=\left|\omega_{C_{i}}^{-1}(-1)\right|=\left|E_{i}\right|$ for $i=1,2$. If $\left|E_{0}\right| \geqslant n / 3$, then let $\Gamma$ be one of $\left\{\Gamma_{1}, \Gamma_{2}\right\}$ with smaller value $\nu$ and thus $\nu(\Gamma)=$ $\min \left\{\nu\left(\Gamma_{1}\right), \nu\left(\Gamma_{2}\right)\right\}=\min \left\{\left|E_{1}\right|,\left|E_{2}\right|\right\} \leqslant\left|E(C) \backslash E_{0}\right| / 3 \leqslant n / 3$.

If $\left|E_{1}\right| \geqslant n / 3$, then let $f^{\prime}=f-g(\bmod 3)$. Note that $f^{\prime}$ is still a $\mathbb{Z}_{3}$-flow and the difference between $f$ and $f^{\prime}$ can only occur on $C$. Thus $\left(f^{\prime}, g\right)$ is also a nowherezero $\mathbb{Z}_{3} \times \mathbb{Z}_{2}$-flow of $G$. Similarly, let $E_{i}^{\prime}=f^{\prime-1}(i) \cap E(C)$ for $i=0,1,2$. Clearly, $E_{0}^{\prime}=E_{1}, E_{1}^{\prime}=E_{2}$, and $E_{2}^{\prime}=E_{0}$. As above, $G$ has a 4 -signed cycle double cover with $\nu=\min \left\{\left|E_{1}^{\prime}\right|,\left|E_{2}^{\prime}\right|\right\}=\min \left\{\left|E_{0}\right|,\left|E_{2}\right|\right\} \leqslant n / 3$.

If $\left|E_{2}\right| \geqslant n / 3$, then let $f^{\prime \prime}=f+g(\bmod 3)$. As the above argument, $\left(f^{\prime \prime}, g\right)$ is also a nowhere-zero $\mathbb{Z}_{3} \times \mathbb{Z}_{2}$-flow of $G$. Also let $E_{i}^{\prime \prime}=f^{\prime \prime-1}(i) \cap E(C)$ for $i=0,1,2$. Clearly, $E_{0}^{\prime \prime}=E_{2}, E_{1}^{\prime \prime}=E_{0}$, and $E_{2}^{\prime \prime}=E_{1}$. Thus $G$ also has a 4 -signed cycle double cover with $\nu=\min \left\{\left|E_{1}^{\prime \prime}\right|,\left|E_{2}^{\prime \prime}\right|\right\}=\min \left\{\left|E_{0}\right|,\left|E_{1}\right|\right\} \leqslant n / 3$. This completes the proof of the first part.

Next we show that $G$ has a 4 -signed cycle double cover with $\nu \leqslant m / 9$. By Theorem 3 and the Eight-Flow Theorem, the graph $G$ admits a nowhere-zero $\mathbb{Z}_{2}^{3}$-flow, say $f=\left(f_{1}, f_{2}, f_{3}\right)$. Let $C_{i}$ be the even subgraph of $G$ induced by $\operatorname{supp}\left(f_{i}\right)$ for $i=1,2,3$. As observed by Bermond et al. [2], the flow $f$ can be selected such that $\left|E\left(C_{1}\right)\right| \geqslant 2 m / 3$. It is obvious that $C_{1}, C_{2}$, and $C_{3}$ form a cycle cover of $G$, i.e., $E(G)=\cup_{i=1}^{3} E\left(C_{i}\right)$. We define seven subsets of $E(G)$ as follows,

$$
E_{i_{1} i_{2} i_{3}}=\left\{e \in E(G): f(e)=\left(i_{1}, i_{2}, i_{3}\right)\right\} \text {, where }\left(i_{1}, i_{2}, i_{3}\right) \in \mathbb{Z}_{2}^{3} \backslash\{\mathbf{0}\} .
$$

Since $f$ is a nowhere-zero $\mathbb{Z}_{2}^{3}$-flow of $G$, the seven subsets defined above form a partition of $E(G)$. 
We claim that $G$ has a 4 -signed cycle double cover with $\nu=\min \left\{\left|E_{i_{1} i_{2} i_{3}}\right|:\left(i_{1}, i_{2}, i_{3}\right) \in\right.$ $\left.\mathbb{Z}_{2}^{3} \backslash\{\mathbf{0}\}\right\}$. To see this, we define an incidence matrix $M$ in the following way. The rows of $M$ correspond to the seven subsets of edges listed in the following order:

$$
E_{100}, E_{110}, E_{111}, E_{101}, E_{010}, E_{011}, E_{001}
$$

The columns of $M$ correspond to the seven even subgraphs of $G$ listed in the following order:

$$
C_{1}, C_{2}, C_{3}, C_{1} \triangle C_{2}, C_{1} \triangle C_{3}, C_{2} \triangle C_{3}, C_{1} \triangle C_{2} \triangle C_{3} .
$$

Let $M=\left(a_{i j}\right)_{7 \times 7}$, where $a_{i j}=1$ if the $i$ th subset is a subset of edges in the $j$ th even subgraph, otherwise $a_{i j}=0$ for $i, j=1,2, \ldots, 7$. Explicitly,

$$
M=\left(\begin{array}{lllllll}
1 & 0 & 0 & 1 & 1 & 0 & 1 \\
1 & 1 & 0 & 0 & 1 & 1 & 0 \\
1 & 1 & 1 & 0 & 0 & 0 & 1 \\
1 & 0 & 1 & 1 & 0 & 1 & 0 \\
0 & 1 & 0 & 1 & 0 & 1 & 1 \\
0 & 1 & 1 & 1 & 1 & 0 & 0 \\
0 & 0 & 1 & 0 & 1 & 1 & 1
\end{array}\right)
$$

It is easily verified that $M$ has the following property:

Each row of $M$ has exactly 4 non-zero entries and each of the other six rows has exactly 2 non-zero entries at the 4 columns corresponding to such 4 non-zero entries of that row.

Let $E^{*}$ be one of the seven subsets of edges with minimum size. Without loss of generality, we may assume that $E^{*}=E_{001}$, i.e., the 7 th subset. Select the four even subgraphs corresponding to the four non-zero entries of the 7 th row, i.e., $C_{3}, C_{1} \triangle C_{3}$, $C_{2} \triangle C_{3}$, and $C_{1} \triangle C_{2} \triangle C_{3}$. By the above property of $M$, these four even subgraphs form a cycle cover of $G$ which covers every edge of $E_{001} 4$ times and all the other edges twice. Replacing all edges of $E_{001}$ in $C_{3}$ by negative edges, we get a 4 -signed cycle double cover with $\nu=\left|E^{*}\right| \leqslant \min \left\{\left|E_{010}\right|,\left|E_{011}\right|,\left|E_{001}\right|\right\} \leqslant\left(m-\left|E\left(C_{1}\right)\right|\right) / 3 \leqslant m / 9$. This completes the proof of the second part.

Proof of Theorem 11. Theorem 3 implies that a graph admitting a nowhere-zero 4-flow has a nowhere-zero $\mathbb{Z}_{2}^{2}$-flow which can obviously be viewed as a nonsurjective $\mathbb{Z}_{2}^{3}$-flow. Conversely, if $G$ admits a nonsurjective nowhere-zero $\mathbb{Z}_{2}^{3}$-flow, then $G$ has a 4 -signed cycle double cover without negative edges by the proof of Theorem 10, i.e., $G$ has a 4 -cycle double cover, which is equivalent to the fact that $G$ admits a nowhere-zero 4-flow by Theorem 9 .

Proof of Theorem 12. Consider the Petersen graph $P$ of order 10 and size 15 . Since $P$ fails to admit a nowhere-zero 4-flow, it has no 4-cycle double cover by Theorem 9 . Thus every 4-signed cycle double cover of $P$ has at least one negative edge. In fact, the lower bound can be achieved, see Fig. 1 for instance: The dotted edge is negative and the first 


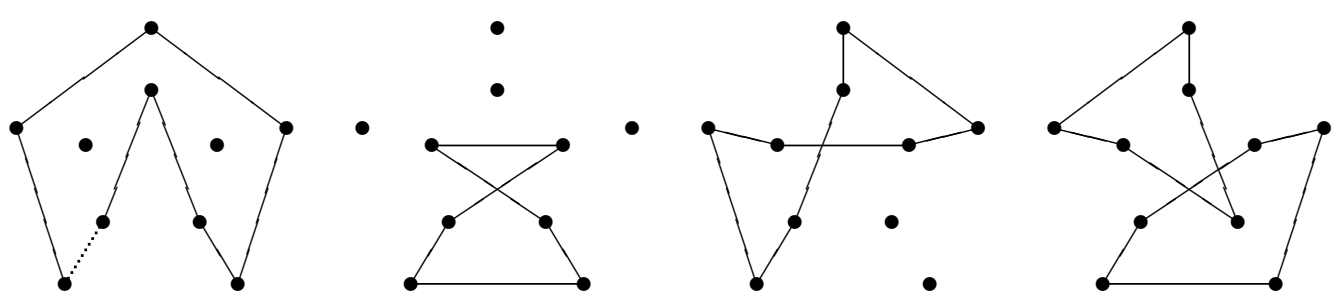

Figure 1: A 4-signed cycle double cover of the Petersen graph.

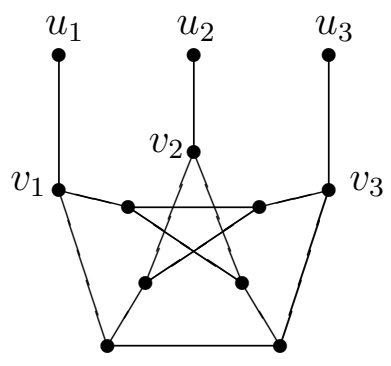

Figure 2: The graph $P^{\prime}$ obtained from the Petersen graph.

even signed graph together with other three even subgraphs shown in Fig. 1 form a 4 signed cycle double cover of $P$ with only one negative edge. For a natural number $k$, a $k$-subdivision of a graph is obtained from the graph by subdividing each edge exactly $k$ times. It is easy to see that a $k$-subdivision of the Petersen graph also has a corresponding 4 -signed cycle double cover with only $k$ negative edges. As in [14], one can construct a more general graph of size $m$ such that its 4-signed cycle double covers all have at least $m / 15$ negative edges. Denote by $P^{\prime}$ the graph obtained from the Petersen graph $P$ in the following way: Delete a vertex $v$ of $P$ and the three edges $v v_{1}, v v_{2}$, and $v v_{3}$ which are incident with $v$, and add three new vertices $u_{1}, u_{2}, u_{3}$ which are called the arms of $P^{\prime}$ and three new edges $u_{i} v_{i}$ for $i=1,2,3$, see Fig. 2. Take a bridgeless cubic graph $G$ of order $n$. A graph denoted by $G \otimes P$, is constructed from $G$ and $P$ as follows: Replace every vertex $v$ of $G$ by a copy of $P^{\prime}$, denoted by $P_{v}^{\prime}$. For every edge $u v$ of $G$, select an arm of $P_{u}^{\prime}$ which is not used and such a arm of $P_{v}^{\prime}$, and identify these two arms forming a new vertex of degree two. It is clear that $G \otimes P$ is also bridgeless. Select a 4-signed cycle double cover of $G \otimes P$ arbitrarily (the existence of such a cover is ensured by Theorem 10), say $\Gamma=\left\{C_{1}, C_{2}, C_{3}, C_{4}\right\}$. For every subgraph $P_{v}^{\prime}$ of $G \otimes P$, identifying its three arms results in a graph isomorphic to $P$, and the collection $\Gamma_{v}=\left\{C_{1} \cap P_{v}^{\prime}, \ldots, C_{4} \cap P_{v}^{\prime}\right\}$ corresponds to a 4 -signed cycle double cover of the graph. Thus $\nu\left(\Gamma_{v}\right) \geqslant 1$ and

$$
\nu(\Gamma)=\sum_{v \in V(G)} \nu\left(\Gamma_{v}\right) \geqslant n=|E(G \otimes P)| / 15,
$$

since the graph $G \otimes P$ has exactly $15 n$ edges. 


\section{Related conjectures}

In this section, we relate the signed cycle double covers to the short cycle covers. The length of a cycle cover is the sum of the lengths of all its cycles. A cycle cover of a graph $G$ with the shortest total length is called the shortest cycle cover of $G$ and the length is denoted by $S C C(G)$. Especially, the length of the shortest 3-cycle cover of $G$ is denoted by $S C C_{3}(G)$. Jaeger (see [17]) made the following general conjecture on the shortest cycle covers.

Conjecture 14. (The Shortest Cycle Cover Conjecture) Every bridgeless graph of size $m$ has a cycle cover with total length at most $7 m / 5$.

The bound in Conjecture 14, if true, is sharp by the Petersen graph. Jamshy and Tarsi [14] showed that Conjecture 14 implies the Cycle Double Cover Conjecture. It is well known that every shortest cycle cover of the Petersen graph consists of 4 even subgraphs, see [9, 14]. Moreover, Fan and Raspaud [7] pointed out that there are infinitely many bridgeless graphs of size $m$ that have no 3-cycle cover with total length less than $22 m / 15$. Tarsi (see [26]) proposed the following conjecture which is weaker than the Berge-Fulkerson Conjecture, see [7].

Conjecture 15. [26] Every bridgeless graph of size $m$ has a 3-cycle cover with length at most $22 m / 15$.

Alon and Tarsi [1] and Bermond, Jackson, and Jaeger [2] independently gave a general upper bound, showing $S C C_{3}(G) \leqslant 5 \mathrm{~m} / 3$ for a bridgeless graph $G$ of size $m$ by applying the Six-Flow Theorem and the Eight-Flow Theorem respectively. Moreover, if $G$ is cubic, then Jackson [10] gave a better bound by $S C C_{3}(G) \leqslant 64 m / 39 \approx 1.641 m$, Fan [6] later improved this bound to $S C C_{3}(G) \leqslant 44 m / 27 \approx 1.629 \mathrm{~m}$, and Král et al. [16] further improved to $S C C_{3}(G) \leqslant 34 m / 21 \approx 1.619 m$. Kaiser et al. [15] extended the Fan bound $S C C_{3}(G) \leqslant 44 m / 27$ from bridgeless cubic graphs to all bridgeless graphs with minimum degree at least 3 .

The short cycle covers also relate to the flow theory. Jamshy et al. [13] proved that the Tutte Five-Flow Conjecture (Conjecture 4) implies the bound $S C C_{3}(G) \leqslant 8 m / 5$ for all bridgeless graphs $G$ of size $m$. Fan [6] proved that the Tutte Three-Flow Conjecture (Conjecture 6) implies that $S C C_{3}(G) \leqslant 92 \mathrm{~m} / 57 \approx 1.614 \mathrm{~m}$. We establish the relationship of the short cycle covers and the signed cycle covers in the following theorem.

Theorem 16. The following statements hold for all graphs $G$ of size $m$.

1. If $G$ has a 4-signed cycle double cover with $\nu$ negative edges, then $G$ has a 3-cycle cover of length at most $3(m+\nu) / 2$.

2. If $G$ has a 3-cycle cover of length $l$, then $G$ has a 4-signed cycle double cover with at most $(l-m) / 5$ negative edges. 
Proof. (1) Let $\Gamma$ be a 4 -signed cycle double cover of $G$ with $\nu$ negative edges. Replacing all the negative edges in $\Gamma$ by normal edges, we get a cycle cover, denoted by $\left\{C_{1}, C_{2}, C_{3}, C_{4}\right\}$, which covers all the negative edges of $\Gamma$ exactly 4 times and the other edges exactly twice. The total length of this cycle cover is $4 \nu+2(m-\nu)=2 m+2 \nu$. Without loss of generality, we assume that $C_{1}$ has the maximum length among the cycle cover, and then the other three even subgraphs $C_{2}, C_{3}$, and $C_{4}$ make up a cycle cover with length no more than $3(m+\nu) / 2$.

(2) Let $\left\{C_{1}, C_{2}, C_{3}\right\}$ be a 3 -cycle cover of $G$ with length $l$. Clearly, $G$ admits a nowherezero $\mathbb{Z}_{2}^{3}$-flow, say $f=\left(f_{1}, f_{2}, f_{3}\right)$, such that $C_{i}$ is induced by $\operatorname{supp}\left(f_{i}\right)$ for $i=1,2,3$. Following the notation such as the edge subsets $E_{i_{1} i_{2} i_{3}}$ in the proof of Theorem 10, we have

$$
l=\sum_{i=1}^{3}\left|E\left(C_{i}\right)\right|=|E(G)|+\left|E_{110}\right|+\left|E_{101}\right|+\left|E_{011}\right|+2\left|E_{111}\right| .
$$

Thus $\left|E_{110}\right|+\left|E_{101}\right|+\left|E_{011}\right|+2\left|E_{111}\right|=l-m$. As in the proof of Theorem 10, the graph $G$ has a 4 -signed cycle double cover with $\nu \leqslant \min \left\{\left|E_{110}\right|,\left|E_{101}\right|,\left|E_{011}\right|,\left|E_{111}\right|\right\} \leqslant$ $(l-m) / 5$.

Theorems 10 and 16 readily imply the bound of Alon-Tarsi and Bermond-JacksonJaeger that $S C C_{3}(G) \leqslant 5 m / 3$ for every bridgeless graph $G$ of size $m$. Theorem 16 also implies the following results.

Corollary 17. Conjecture 13 implies that every bridgeless graph of size $m$ has a 3-cycle cover with length at most $8 \mathrm{~m} / 5$.

Corollary 18. Conjecture 15 implies that every bridgeless graph of size $m$ has a 4-signed cycle double cover with at most $7 \mathrm{~m} / 75$ negative edges.

Corollary 17, compared with the result of Jamshy et al. [13], indicates that it might be hard to prove Conjecture 13, and Corollary 18 shows that the bound in Theorem 10 might also be hardly improved since the bound $7 \mathrm{~m} / 75$ is slightly better than $\mathrm{m} / 9$.

\section{References}

[1] N. Alon and M. Tarsi. Covering multigraphs by simple circuits. Algebraic Discrete Methods, 6:345-350, 1985.

[2] J. Bermond, B. Jackson, and F. Jaeger. Shortest coverings of graphs with cycles. J. Combin. Theory, Ser. B, 35:297-308, 1983.

[3] J. A. Bondy and U. S. R. Murty. Graph Theory. Springer, 2008.

[4] J. A. Bondy and U. S. R. Murty. Graph Theory with Applications. Macmillan, London, 1976.

[5] U. A. Celmins. On cubic graphs that do not have an edge 3-coloring. Ph.D. thesis, University of Waterloo, Ontario, Canada, 1984. 
[6] G. Fan. Shortest cycle covers of cubic graphs. J. Graph Theory, 18(2):131-141, 1994.

[7] G. Fan and A. Raspaud. Fulkerson's conjecture and circuits covers. J. Combin. Theory, Ser. B, 61:133-138, 1994.

[8] D. R. Fulkerson. Blocking and anti-blocking pairs of polyhedra. Math. Programming, 1:168-194, 1971.

[9] A. Itai and M. Rodeh. Covering a graph by circuits. InAutomata, Languages and Programming, volume 62 of Lecture Notes in Computer Science, pages 289-299. Berlin: Springer-Verlag, 1978.

[10] B. Jackson. Shortest circuit covers of cubic graphs. J. Combin. Theory, Ser. B, 60:299-307, 1994.

[11] F. Jaeger. Flows and generalized coloring theorems in graphs. J. Combin. Theory, Ser. B, 26:205-216, 1979.

[12] F. Jaeger. On nowhere-zero flows in multigraphs. Proceedings of the Fifth British Combinatorial Conference 1975, Congr. Numer., 15:373-378, 1975.

[13] U. Jamshy, A. Raspaud, and M. Tarsi. Short circuit covers for regular matroids with nowhere-zero 5-flows. J. Combin. Theory, Ser. B, 43:354-357, 1987.

[14] U. Jamshy and M. Tarsi. Shortest cycle covers and the cycle double cover conjecture. J. Combin. Theory, Ser. B, 56:197-204, 1992.

[15] T. Kaiser, D. Král, B. Lidický, and P. Nejedlý. Short cycle covers of graphs with minimum degree three. SIAM J. Discrete Math., 24:330-355, 2010.

[16] D. Král, P. Nejedlý, and R. Šámal. Short cycle covers of cubic graphs. KAMDIMATIA Series 2008-846, Department of Applied Mathematics, Charles University, Prague, Czech, 2008.

[17] E. Máčajová, A. Raspaud, M. Tarsi, and X.-D. Zhu. Short cycle covers of graphs and nowhere-zero flows. J. Graph Theory, 68:340-348, 2011.

[18] E. Máčajová and M. Škoviera. Fano colourings of cubic graphs and the Fulkerson conjecture. Theoretical Computer Science, 349:112-120, 2005.

[19] M. Preissmann. Sur les colorations des arêtes des graphes cubiques. Thèse de Doctorat de $3^{e m e}$, Ph.D. thesis, Université de Grenoble, France, 1981.

[20] P. D. Seymour. Nowhere-zero 6-flows. J. Combin. Theory, Ser. B, 30:130-135, 1981.

[21] P. D. Seymour. On multi-colourings of cubic graphs, and conjectures of Fulkerson and Tutte. Proc. London Math. Soc., 38(3):423-460, 1979.

[22] P. D. Seymour. Sums of circuits. In Graph Theory and Related Topics, (J. A. Bondy and U. S. R. Murty, eds.) pages 341-355, Academic Press, New York, 1979.

[23] G. Szekeres. Polyhedral decompositions of cubic graphs. Bull. Austral. Math. Soc., 8:367-387, 1973.

[24] W. T. Tutte. A contribution to the theory of chromatic polynomials. Canadian J. Math., 6:80-91, 1954. 
[25] W. T. Tutte. On the algebraic theory of graph colorings. J. Combin. Theory, 1:15-50, 1966.

[26] C. Zhang. Circuit Double Covers of Graphs. Cambridge University Press, 2012.

[27] C. Zhang. Integer flows and cycle covers of graphs. Marcel Dekker, New York, 1997. 